\title{
La reanimación cardiopulmonar desde la experiencia del estudiante: estudio de caso*
}

\author{
Cardiopulmonary Resuscitation Experience from Student: Case Study \\ Ressuscitação cardiopulmonar desde a experiência do estudante: estudo de caso
}

\author{
Mery Luz Valderrama Sanabria ${ }^{\text {a }}$ \\ Universidad de La Sabana, Colombia \\ Universidad de los Llanos, Colombia \\ mvalderrama@unillanos.edu.co
}

Ruth Natalia Riaño Niño

Universidad Pedagógica y Tecnológica de Colombia

(UPTC), Colombia
DOI: https://doi.org/10.11144/Javeriana.ie20-1.rcee

Fecha de recepción: 14 Mayo 2016 Fecha de aprobación: 16 Noviembre 2016

\section{Resumen:}

Introducción: la reanimación es un momento de cuidado que en el profesional de enfermería genera sentimientos y emociones para los cuales no se está preparado, debido a que la formación está centrada en el componente teórico-práctico. Esta situación es similar a la del estudiante. Objetivo: describir la experiencia del estudiante durante la reanimación cardiopulmonar, a fin de reconocer aspectos esenciales para la formación profesional. Materiales y método: abordaje cualitativo, fenomenológico, mediante un estudio de caso. Para recolectar y analizar la información se utilizó la entrevista semiestructurada y la observación, que se registró en notas de campo. Resultados: las categorías que surgieron del análisis se denominaron: conciencia de la subjetividad, apoyo del docente, cuestionamiento frente a la cotidianidad de la muerte en el equipo de salud, la vida como motivación y el resignificado de la muerte. Se contrastaron los resultados de la investigación a la luz de la literatura. Conclusiones: el estudiante experimenta vivencias en las cuales aflora la subjetividad; la muerte es una de ellas y tiene mayor significado cuando se asume por primera vez, puesto que no se está preparado para abordarla. Ayudar a morir con dignidad y acompañar en el sufrimiento requiere formación, conocimiento y madurez, en el futuro profesional, dada la implicación emocional que genera. Por tanto, se deben propiciar espacios de reflexión en torno a estas situaciones críticas, preferiblemente desde las experiencias de los mismos estudiantes, para fortalecer los aspectos que hacen de esta una experiencia enriquecedora.

Palabras clave: estudios de caso, muerte, resucitación cardiopulmonar, enfermería de urgencia.

\section{Abstract:}

Introduction: Cardiopulmonary CPR resuscitation is a moment of care generated in the professional nursing feelings and emotions for which you are not prepared, because the training is focused on the theoretical and practical component; this situation is similar to the student. Aim: To describe the student experience during CPR, to recognize essential aspects for vocational training. Materials and Methods: A qualitative approach, phenomenological, through a case study. To collect and analyze information through semistructured interviews and observation was recorded in field notes was used. Results: The categories that emerged from the analysis were named: Awareness of subjectivity, teacher support, questioning face death every day in the health team, life as motivation and redefined death. The results of research in the light of the literature were contrasted. Conclusions: The student experiences where subjectivity comes out, death is one of them and has more meaning when it is assumed for the first time, since you are not prepared to address it. Helping to die with dignity and accompany suffering requires training, knowledge and maturity in the professional future, given the emotional involvement it generates. Therefore, should promote opportunities for reflection on these critical situations, preferably from the experiences of these students in order to strengthen the aspects that make this an enriching experience.

Keywords: case studies, death, cardiopulmonary resuscitation, emergency nursing.

\section{Resumo:}

Introdução: a ressuscitação cardiopulmonar (RCP) é um momento de cuidado que no professional de enfermagem gera sentimentos e emoções para as quais não está preparado, porque o treinamento é focado na teória e na prática. Objetivo: descrever a experiência do estudante durante a RCP, para reconhecer aspectos essenciais para a formação profissional. Materiais e métodos: abordagem qualitativa, fenomenológica, através de um estudo de caso. Para recolher e analisar a informação, nós usamos entrevistas Notas de autor 
e observação semi-estruturada que foi gravada em notas de campo. Resultados: as categorias que emergiram da análise foram nomeados: a consciência da subjetividade, apoio dos professores, questionando diante a cotidianidade da morte na equipe de saúde, a vida como motivação e o entendimento da morte. Os resultados da pesquisa foram comparados à luz da literatura. Conclusões: o estudante tem experiências onde a subjetividade emerge, a morte é uma delas e tem mais significado quando e assumida pela primeira vez, porque a pessoa não está preparada para enfrentá-lo. Ajudar a morrer com dignidade e acompanhar no sofrimento que exige formação, conhecimento e maturidade, no futuro profissional, dado o envolvimento emocional que ele gera. Portanto, ele deve promover espaços para a reflexão sobre estas situações críticas, de preferência a partir das experiências dos próprios alunos, a fim de reforçar os aspectos que tornam esta uma experiência enriquecedora.

Palavras-chave: estudo de casos, morte, ressuscitação cardiopulmonar, enfermagem de emergência.

\section{Introducción}

La reanimación cardiopulmonar es un momento de cuidado que en el profesional de enfermería genera sentimientos y emociones para los cuales, en ocasiones, no está preparado, debido a que la formación está centrada en el componente teórico-práctico. Esta situación es similar a la del estudiante; por lo tanto, es fundamental describir esta experiencia, a fin de reconocer aspectos esenciales para la formación profesional.

De acuerdo con la revisión de la literatura sobre el tema, en ella se menciona la formación integral del futuro profesional de enfermería; sin embargo, el énfasis sigue estando en torno a la dimensión científico-técnica y se deja de lado el análisis de la percepción y los sentimientos de los estudiantes, en cuanto a su formación como persona en el ámbito de la enfermería. A su vez, se ha observado que este proceso de enseñanza-aprendizaje no tiene en cuenta aspectos que permitan el fortalecimiento emocional de los futuros profesionales [1].

En este sentido, algunos estudiantes de enfermería que experimentan ansiedad, junto con momentos emotivos, vivenciada durante las prácticas clínicas, están en riesgo de abandonar la carrera y se convierte en un factor de deserción universitaria, por la carga emocional que se vive [2]. En consecuencia, se deben conocer los sentimientos y las percepciones que experimentan los estudiantes al afrontar estas vivencias significativas por primera vez, al igual que los mecanismos de afrontamiento que adoptan los profesionales [2], puesto que las emociones no son ajenas a la esencia de la enfermera; al contrario, requieren una presencia concreta, explícita, honesta y real para poder comprender e interpretar la esencia del cuidado [3].

Otra consideración para tener en cuenta es la edad de los estudiantes cuando ingresan a la universidad, cada vez más temprana. Muchos de ellos se encuentran en plena adolescencia y, por lo tanto, en un periodo de desarrollo importante y particular en sus vidas. Además, suelen llegar bajo estrés y presión severa, con una serie expectativas y deseos que aumentan la carga emocional. A veces, muchos se enfrentan con una realidad diferente a la esperada, tanto en relación con las exigencias académicas como con las condiciones de la enseñanza; entonces se perciben frente a una nueva etapa de sus vidas, en la que se acumulan sentimientos y emociones. Asimismo, sus experiencias influyen en los pensamientos y acciones, de tal manera que la humanización durante la formación del futuro profesional requerirá una nueva actitud por parte de los educadores que contemple a la persona (estudiante) de manera integral, puesto que se menciona que los profesores hablan mucho sobre el holismo; sin embargo, este enfoque no es experimentado por ellos a lo largo de la formación [1].

Por otro lado, la fundamentación teórica del acto de cuidado se basa en diferentes teorías y modelos de formación, impulsados desde la misión y la visión de cada institución formadora, acorde con la estructura curricular [4]. En tal sentido, para que el profesional de enfermería pueda actuar con seguridad y garantizar la sobrevida del paciente debe tener la preparación, el conocimiento y la actualización sobre las maniobras de reanimación [5]. Además, el adecuado aprendizaje y posterior implementación de un buen abordaje en la reanimación cardiocerebropulmonar de la persona que presenta paro cardiorrespiratorio, mejora el pronóstico [5,6,7].

En la actualidad, gracias a tecnologías como los simuladores de alta fidelidad, se han dado nuevas posibilidades en la enseñanza y el aprendizaje para enfermería. Su uso prepara a los estudiantes a situaciones 
semejantes a las que enfrentarán en la realidad del campo profesional; la simulación les presenta problemas sobre los cuales deben intervenir, a fin de favorecer el desarrollo de habilidades de índole cognitivo, motriz y actitudinal, y se convierte en una herramienta que apoya los procesos de formación, al promover la reflexión ante la experiencia de los alumnos, en la mediación que realiza el docente, a través del diseño de escenarios de aprendizaje, su ejecución y evaluación. No obstante, su empleo no sustituye la práctica en los escenarios reales de desempeño profesional [8].

\section{Materiales y métodos}

Esta investigación tuvo un abordaje de tipo cualitativo, fenomenológico y descriptivo [9], por medio de un estudio de caso instrumental, que Stake [10] define como aquel que el investigador elige por ser el prototipo y facilitar la ilustración de un tema o situación específica. Los estudios de caso se refieren a una única unidad muestral, bien sea una persona, grupo u organización [11]. Para la selección del caso hubo una reunión con diez estudiantes que en su práctica clínica vivenciaron el cuidado a una persona con necesidad de reanimación cardiopulmonar. Se les explicó el objetivo del estudio y la garantía de la privacidad de la información suministrada. Cinco aceptaron participar, previa firma del consentimiento informado. Cada uno narró su experiencia para ser grabada y transcrita; luego se seleccionó la de mayor contenido, calidad y profundidad. Se tuvo en cuenta que la experiencia fuera reciente, que el estudiante hubiera presenciado la situación y que la evaluara como enriquecedora para su formación.

Para recolectar información que complementara el caso escogido se realizaron varias entrevistas a profundidad, en las cuales se procuró esclarecer la experiencia humana subjetiva. La duración de las entrevistas fue de cuarenta a sesenta minutos. En todos los casos, el participante mostró buena disposición para su desarrollo. La entrevista inicial partió de una pregunta central, desde la cual se estructuró el orden y la pertinencia de las siguientes. Se tuvo en cuenta la autonomía del participante para dar por terminada la entrevista en cualquier momento [12].

Se realizaron tres entrevistas que se grabaron y transcribieron en su totalidad. Algunas expresiones no verbales (gesticulaciones del rostro o movimientos de las manos) observadas también se escribieron y registraron en el diario de campo de la investigación. Estas expresiones arrojaron datos importantes que no fueron posibles de identificar a través de la entrevista [13].

El primer paso del análisis de la información recolectada fue transcribir fielmente las entrevistas, sin omitir las frases y expresiones que generó el participante dentro de su contexto; después se compararon con la grabación inmediatamente terminada. Luego se realizó la inmersión en los datos, se leyeron línea por línea cada una de las entrevistas [12], se establecieron descriptores, los cuales se agruparon en códigos y, posteriormente, en categorías que describen la experiencia.

Para asegurar el rigor y la validez de la investigación, las autoras del trabajo y un asesor externo realizaron la triangulación de investigadores durante la fase inicial de la codificación mediante una confirmación de manera independiente. Los resultados finales con categorías y subcategorías identificadas se pusieron a consideración del participante, para verificar si los reconocía como verdaderos. De esta manera se garantizó la credibilidad de los resultados y las conclusiones del estudio [14].

Los aspectos éticos que se tuvieron en cuenta fueron: consentimiento informado, confidencialidad, privacidad de identidad mediante el uso de códigos o etiquetas, respeto a la autonomía y lo establecido en Resolución 008430 del Ministerio de Salud de Colombia. 
Resultados

La experiencia ocurrió en el servicio de urgencias de una institución de salud de tercer nivel, en la práctica de séptimo semestre de enfermería. Durante el turno, la estudiante participó en la reanimación de un adulto mayor que ingresó en paro cardiorrespiratorio. A continuación se presentan las categorías que emergen del análisis de contenido.

\section{Conciencia de la subjetividad}

Cuando el estudiante de enfermería interactúa con el sujeto de cuidado, se entremezclan emociones y respuestas humanas que son expresadas de diversas formas. En tal sentido, esta categoría se describe a partir de los recursos propios del futuro enfermero(a), que le permitieron asumir la reanimación cardiopulmonar como una oportunidad de aprendizaje. A continuación se presentan las subcategorías que surgieron en el estudio:

Confianza en sí mismo: "... nosotros nos ofrecimos para continuar con el proceso y empecé yo. Se me subió la adrenalina e inmediatamente me lancé, porque más que hacer y poner en práctica lo que le enseñan a uno en cada clase, yo estaba pensando en eso que puedo hacer para que la persona viva. Pensé 'puedo y quiero hacerlo, puedo lograr algo"” [E2P1].

Sentimientos y emociones: “... inicié haciendo compresiones torácicas. Durante el primer ciclo, hubo miedo; cuando se volvió a valorar el pulso, fueron cambiando mis sentimientos, porque no había respuesta. Me empecé a afanar, agotar más de la cuenta, uno se cansa, pero con el nerviosismo, angustia, miedo, impotencia, de que la persona no estaba respondiendo; me empecé a estresar un poquito, sentía que podía continuar con un siguiente ciclo, pero decidí que otra persona entrara, fue una compañera, por ese mismo sentimiento que me estaba generando" [E1P3].

Los sentimientos y las emociones experimentados por la estudiante fueron manifestados de manera diversa: se alternaron el miedo con la impotencia, al ponerse en la piel de otro.

Creencia en un ser superior: "Mucho después de esa práctica, una vez soñé con lo sucedido, y al otro día en algún momento se me vino a la mente lo que había pasado, de pronto lo había mencionado antes, quiero reiterar la parte de encomendarnos a Dios en todo lo que hagamos así sea mentalmente, que cada compresión, sea dirigida por él, cada ventilación, todo por él y encomendarnos absolutamente a él” [E3P4].

Esta subcategoría pone de manifiesto un grado muy elevado de implicación emocional del futuro profesional en el cuidado del paciente moribundo; sin embargo, se conserva la fe en un ser superior para encomendarle cada acto de cuidado.

\section{Apoyo del docente}

Esta categoría hace referencia a la influencia que en este caso ejerce el docente para que la estudiante se motive a participar en el acto de cuidado. Ella identificó que la confianza fue determinante para considerar su intervención útil, a pesar de la duda que generó enfrentarse por primera vez a esta situación:

Cuando el profesor me invita a participar en la reanimación, a mí realmente me dio mucha confianza, me sentí útil en un proceso del cual se requería la mayor ayuda posible buscando un fin o beneficio para esa persona y realmente sentí que lo que él me estaba diciendo era porque de alguna forma, yo le había demostrado confianza en lo que podía hacer y me dio esa capacidad de creer en mí misma, porque no voy a negarlo, en el momento sentí, ¿qué tal lo haga mal? [E1P5] 


\section{Cuestionamiento frente a la cotidianidad de la muerte en el equipo de salud}

Al ser la primera vez que la estudiante se enfrenta a la muerte, surgen sentimientos de tristeza, impotencia, temor, entre otros, que propician la reflexión frente a la situación y asumir que el personal de salud lo está afrontando de la misma manera; sin embargo, ella observa actitudes que la llevan a cuestionarse acerca de la cotidianidad de la muerte y si existe la posibilidad de asumirla como habitual en el futuro ejercicio profesional:

No se les ve o no se sabe interiormente cómo les ha afectado esa situación, pero así, de manera exterior, se veía como cualquier cosa que se ve a diario, eso también me dolió. Por ejemplo, él falleció, quedó con los ojos abiertos, con la boca abierta. No sé, al menos pensé "tápenlo, no lo dejen así”. Inclusive fuimos nosotras que decidimos hacer eso, le cerramos los ojos, lo acomodamos, con una sábana le tapamos la cara y el torso porque lo dejaron ahí y se fueron hacer sus actividades. No sé cómo es el protocolo, pero eso también me generó impacto y en algún momento pensé “ ¿será que nosotros como estudiantes llegará el momento en que no nos dejemos invadir por esas situaciones y demostremos como tanta frialdad ante ese hecho?”. [E2P6]

\section{La vida como motivación}

Se considera la vida como objetivo de la intervención, el deseo de hacer lo mejor posible por la persona, en términos de supervivencia, sin contemplar la opción de la muerte:

Uno se comienza a centrar más que todo en poder hacer lo que más pueda, casi no en cómo me lo enseñaron en clase, sino aplicar todo de una vez y se centra más en poder salvar a la persona o brindarle bienestar y a medida que iba pasando el tiempo eran esos sentimientos los que se veían floreciendo que a pesar de lo que hice, de lo que había aprendido y que lo hicimos bien, porque sabemos que lo hicimos bien, no se logró el objetivo por el cual uno lo hace, por eso yo creo que nos sentimos desanimadas y tristes, además de ver una persona morirse en ese momento. [E3P1]

\section{Resignificado de la muerte}

Aunque la motivación es mantener con vida a la persona, cuando la estudiante se enfrenta a la posibilidad de la muerte, se genera un proceso de reflexión frente al significado de esta; la considera una circunstancia real que debe ser tenida en cuenta durante el proceso de formación y en el ejercicio profesional. La muerte es el fin inevitable de la vida humana, y en la sociedad actual se habla muy poco de ella, de ahí que la futura enfermera requiera entender que es algo natural y cotidiano al ejercicio profesional:

Esta experiencia también nos abrió la posibilidad de sentarnos a reflexionar que la muerte es un proceso natural y como estudiantes y futuros profesionales ya es algo a lo que nos vamos a tener que ver enfrentados y hay que empezar ese proceso de asimilar esa situación y dejar un poquito al lado esa sorpresa que de pronto le puede generar a uno enfrentarse a estas circunstancias. [E1P7]

\section{Discusión}

Al contrastar los resultados de la investigación con la literatura sobre el tema, se obtuvo que en la categoría conciencia de la subjetividad, de las situaciones vividas con más intensidad por los estudiantes de enfermería en la práctica clínica se encuentran el paro cardiorrespiratorio y la muerte de la persona, pues allí se experimentan sentimientos de impotencia, incertidumbre y, muchas veces, temor de causar daño [15].

A pesar de lo anterior, la estudiante moviliza la subjetividad y decide participar en el cuidado de esta persona, gracias a recursos propios como la confianza en sí misma. Así como la estudiante "se pone en la piel del otro" [2], la emoción parece ser la mayor amenaza para su imagen, lo que facilita la expresión de sentimientos, que le permiten esclarecer la realidad interna necesaria en el proceso de formación del futuro profesional de enfermería $[1,16,17]$. Cuartas menciona: 
Cierta vez alguien me dijo que admiraba a la enfermera porque era quien estaba cara a cara con sus semejantes en las situaciones más miserables y paupérrimas de su existencia. Y es justamente eso lo que me hace pensar si como profesionales no necesitaríamos inventarnos una especie de armadura para no derrumbarnos ante situaciones tan complejas y tristes. Será necesario forrarse el cuerpo, el corazón y el alma para ofrecer el mejor cuidado sin que esto nos afecte. Pero, de igual forma, esta armadura deberá tener ciertas características especiales: deberá ser fácil de poner y quitar, pues una de las cosas que más temo de pertenecer a esta profesión es precisamente que me anestesie frente al dolor del otro. [18]

Por su parte, el apoyo que el profesor le brinda a la estudiante durante la práctica, facilita el aprendizaje, pues le da seguridad, como se menciona en el estudio de caso. Asimismo, contribuye a disminuir temores y angustias; el docente se convierte en alguien en quien confiar, dados los conocimientos y experiencia que posee [19]. Su misión es fomentar la toma de decisiones y la capacidad de resolver problemas de forma independiente [20]. Debe reconocerse la posición empática por parte del docente para ayudar a cada estudiante y considerar sus sentimientos [1]. En tal sentido, le corresponde formular estrategias que conduzcan a fomentar las capacidades de afrontamiento de los futuros profesionales ante situaciones en las cuales se movilizan emociones para las que no se está preparado [2,19]. El profesor ha de promover la motivación en sus estudiantes, para que surja en ellos la iniciativa, como ocurrió en la investigación, cuando la participante menciona "yo me lance", para conocer y aplicar los conocimientos.

De esta forma, la estudiante se convierte en protagonista de su propia aventura de aprendizaje [21], y esto también concuerda con los planteamientos de Benner, en su Modelo de Aprendiz a Experto, en el que explica que la transición del enfermero novato a experto requiere experiencia e involucrarse emocionalmente con el paciente y su familia [2].

En la categoría cuestionamiento frente a la cotidianidad de la muerte en el equipo de salud, la participante no comprende en qué magnitud haya afectado emocionalmente al personal de salud el hecho ocurrido. En tal sentido, Henao, Fajardo y Núñez [2] consideran que el estudiante de enfermería hace consciente su falta de "experiencia” y reconoce que con el tiempo y la práctica logrará adquirir las destrezas que le permitan reducir las sensaciones desagradables que emergen de los actos de cuidado. A su vez, Hanzeliková y colaboradores [17] mencionan que el trato que recibe el moribundo por parte de los profesionales de salud está condicionado por la forma en que entienden el fenómeno y proceso de la muerte; por tanto, la falta o escasa preparación puede llevar a producir ciertas alteraciones en la esfera emocional de los profesionales.

En la categoría la vida como motivación, la literatura menciona que enfrentar la muerte es una experiencia dolorosa y traumática que puede generar sentimientos de desconsuelo, rechazo y negación que, a la vez, motiva el cuestionamiento acerca del quehacer en enfermería. Además, tener la experiencia de reanimar a una persona compromete la parte emocional del estudiante, más cuando el desenlace es la muerte [21]. Lo anterior se evidenció en el estudio de caso, cuando la participante menciona el interés por hacer todo lo posible para salvar a la persona. Asimismo, compadecerse de alguien no significa asumir la autonomía del otro, sino ponerse en su lugar y promover el desarrollo de la autonomía ajena; por ello es pertinente abordar con los futuros profesionales la necesidad de establecer estrategias de aprendizaje que contribuyan a realizar una práctica de enfermería en la que los sentimientos se conviertan en momentos de cuidado enriquecedores para la formación [2].

Por otra parte, una vez la estudiante se da cuenta de que el objetivo propuesto no va ser posible, resignifica el concepto de la muerte. Con relación a esta categoría, la literatura menciona que se debe hacer un análisis luego de ocurrida la situación, con el fin de aprender a asimilar y a manejar lo inevitable, e ir aceptando la realidad, para facilitar que el estudiante contemple que la muerte es algo que acompaña el ejercicio profesional y que hay que contemplarla como una posibilidad real [22].

Lopes Magalhães y Lyra da Silva mencionan que, en el momento de cuidar, algunos profesionales de enfermería tienen dificultad en sus imaginarios para tratar con los conceptos de muerte y morir, principalmente cuando involucran valores culturales y creencias religiosas [23]. Lo anterior se evidenció en la investigación cuando la estudiante interpreta que la muerte para el personal de salud es un hecho cotidiano. 
La muerte es la etapa más segura desde el momento que se empieza a vivir; pero la que menos se espera y se sabe afrontar o entender; además, no se enseña a los estudiantes a conocer, entender, enfrentar y manejar la muerte más allá del discurso teórico [3]. Para el profesional de enfermería, el encuentro diario con la muerte y con el sufrimiento genera una inmensa carga de emociones, y desenvolverse en situaciones sin implicarse emocionalmente, pero sin parecer frívolos, es bastante difícil [17].

\section{Conclusiones}

En ocasiones, las vivencias que experimenta el estudiante durante la práctica clínica están rodeadas de sentimientos, afectos y emociones, porque se interactúa con la persona que está a su cuidado. Ahí es donde sale a flote la subjetividad y el estudiante se enfrenta a situaciones reales, para configurar la experiencia, producto de la confrontación de su ser con la realidad.

Para el estudiante, la muerte no deja de ser un acontecimiento dramático para el que no se siente preparado; por tanto, se requiere formación, conocimiento y madurez, en el futuro profesional, dada la implicación emocional que genera. De ahí que sea necesario propiciar espacios de reflexión en torno a estas situaciones críticas, desde las experiencias de los mismos estudiantes, para que fortalezcan los aspectos que hacen de esta una experiencia enriquecedora y para que aprendan a manejar situaciones complejas que faciliten el acercamiento y la empatía y eviten la inestabilidad de emociones en el futuro profesional.

\section{Referencias}

1. Vollrath A, Angelo M, Muñoz L. Vivencia de estudiantes de enfermería de la transición a la práctica profesional: un enfoque fenomenológico social. Rev Texto Contexto Enferm. 2011;20 (Esp):66-73.

2. Henao M, Fajardo E, Núñez M. La experiencia de estudiantes de enfermería ante el dolor infligido en la práctica clínica. Rev Salud Uninorte. 2014;30(3):431-41.

3. García M, Ruiz M, González P, Fernández M, De Blas Gómez I. Experiencias y vivencias del estudiante de $4^{\circ}$ de enfermería en el practicum. Revista Cultura de los Cuidados. 2014;18(38):25-33.

4. Delgado A, Naranjo M. El Acto de cuidado de enfermería como fundamentación del quehacer profesional investigativo. Av Enferm. 2015;33(3):412-9.

5. Olivetto A, Muglia I, Barcellos M, Araujo S. Conocimiento teórico de los enfermeros sobre parada cardiorrespiratoria y resucitación cardiopulmonar en unidades no hospitalarias de atención de urgencia y emergencia. Rev LatinoAm Enfermagem. 2011;19(2):[8 pantallas].

6. Cárdenas D. Análisis de un programa de formación masiva en soporte vital básico para la población general [tesis doctoral]. Granada: Universidad de Granada; 2012.

7. Balcázar-Rincón LE, Mendoza-Solís LA, Ramírez-Alcántara YL. Reanimación cardiopulmonar: nivel de conocimientos entre el personal de un servicio de urgencias. Rev Esp Méd Quir. 2015;20:248-55.

8. Piña I, Amador R. La enseñanza de la enfermería con simuladores, consideraciones teórico-pedagógicas para perfilar un modelo didáctico. Enferm Univ. 2015;12(3):152-59.

9. Espitia EC. La fenomenología interpretativa como alternativa apropiada para estudiar los fenómenos humanos. Invest Educ Enferm. 2013;17:27-35.

10. Stake RE. Investigación con estudios de casos. $5^{\mathrm{a}}$ ed. Madrid: Morata; 2010.

11. Urra Medina E, Núñez Carrasco R, Retamal Valenzuela C, Jure Cares L. Enfoques de estudio de casos en la investigación de enfermería. Cienc Enferm. 2014;1:131-42.

12. Do Padro ML, De Souza M, Cometto MC, Gómez P. Investigación cualitativa en enfermería. Washington: Metodología y Didáctica; 2015.

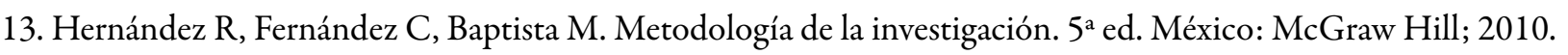


14. Noreña AL, Alcaraz-Moreno N, Rojas JG, Rebolledo-Malpica D. Aplicabilidad de los criterios de rigor y éticos en la investigación cualitativa. Aquichan. 2012;12(3):263-74.

15. Moya Nicolás M Larrosa Sánchez S, López Marín C, et al. Percepción del estrés en los estudiantes de Enfermería ante sus prácticas clínicas. Enferm Glob. 2013;12(31):232.

16. Nunes A, Carneiro L. O cuidado e a clínica na formação do enfermeiro: saberes, práticas e modos de subjetivação. Rev Esc Anna Nery. 2011;15(4):776-83.

17. Hanzeliková Pogrányivá A, García López MV, Conty Serrano MR, et al. Reflexiones de los alumnos de enfermería sobre el proceso de la muerte. Enferm Glob. 2014;33:133-44.

18. Cuartas K. Cinco minutos en la mente de una estudiante de enfermería. Investig Educ Enferm. 2010;28(1):119-21.

19. Hoyos Duque T, et al. La experiencia de los estudiantes de enfermería de la Universidad de Antioquia en la práctica clínica. [tesis de maestría]. Manizales: Universidad de Manizales; 2005.

20. Molina Chailán P, Jara Concha P. El saber práctico en enfermería. Rev Cuba Enferm. 2010;26(2):37-43.

21. Sosa C, Pavia E, Mendoza J. Aptitud clínica de enfermería en pacientes con paro cardiorrespiratorio: alcances de una estrategia educativa. Rev Enferm Inst Mex Seguro Soc. 2009;85-90.

22. Neumann Olave KM. La vivencia del estudiante de enfermería de la universidad austral de chile ante la muerte de sus pacientes, durante su experiencia clínica [tesis de pregrado]. Valdivia: Universidad Austral de Chile; 2009.

23. Lopes Magalháes AV, Lyra da Silva RC. Los conceptos de muerte y morir en enfermería y las relaciones con el ethos del cuidado. Enferm Glob. 2009;17:1-10.

\section{Notas}

* Artículo de investigación.

\section{BY}

\section{Información adicional}

Cómo citar: Valderrama Sanabria ML, Riaño Niño RN. La reanimación cardiopulmonar desde la experiencia del estudiante: estudio de caso. Investig Enferm Imagen Desarr. 2018;20(1):xx-xx. https://doi.org/10.1114 4/Javeriana.ie20-1.rcee 